\section{BRAZIULIAN JOURNAL \\ OF MEDICAL AND BIOLOGICAL RESHARCH}

www.bjournal.com.br
ISSN 0100-879X

Volume 43 (8) 698-811 August 2010

BIOMEDICAL SCIENCES

AND

CLINICAL INVESTIGATION

Braz J Med Biol Res, August 2010, Volume 43(8) 722-727

doi: 10.1590/S0100-879X2010007500064

Gene expression promoted by the SV40 DNA targeting sequence and the hypoxia-responsive element under normoxia and hypoxia

C.B. Sacramento, J.Z. Moraes, P.M.A. Denapolis and S.W. Han

The Brazilian Journal of Medical and Biological Research is partially financed by
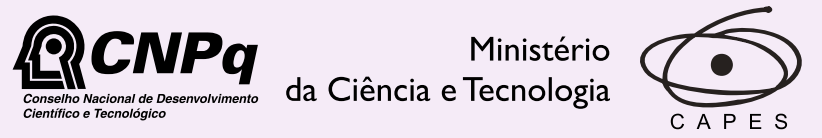

Ministério da Educação

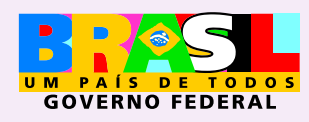

DFAPESP

Institutional Sponsors
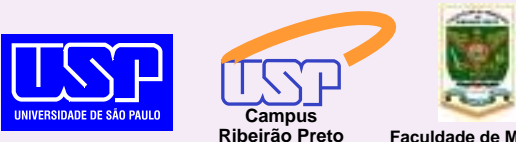

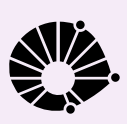

UNICAMP
Ф SHIMADZU

GE Healthcare

Hotsite of proteomics metabolomics developped by: 


\title{
Gene expression promoted by the SV40 DNA targeting sequence and the hypoxia-responsive element under normoxia and hypoxia
}

\author{
C.B. Sacramento ${ }^{1}$, J.Z. Moraes ${ }^{2}$, P.M.A. Denapolis ${ }^{1}$ and S.W. $\operatorname{Han}^{1,2}$ \\ ${ }^{1}$ Centro Interdisciplinar de Terapia Gênica and ${ }^{2}$ Departamento de Biofísica, \\ Universidade Federal de São Paulo, São Paulo, SP, Brasil
}

\begin{abstract}
The main objective of the present study was to find suitable DNA-targeting sequences (DTS) for the construction of plasmid vectors to be used to treat ischemic diseases. The well-known Simian virus 40 nuclear DTS (SV40-DTS) and hypoxia-responsive element (HRE) sequences were used to construct plasmid vectors to express the human vascular endothelial growth factor gene (hVEGF). The rate of plasmid nuclear transport and consequent gene expression under normoxia $\left(20 \% \mathrm{O}_{2}\right)$ and hypoxia (less than $5 \% \mathrm{O}_{2}$ ) were determined. Plasmids containing the SV40-DTS or HRE sequences were constructed and used to transfect the A293T cell line (a human embryonic kidney cell line) in vitro and mouse skeletal muscle cells in vivo. Plasmid transport to the nucleus was monitored by real-time PCR, and the expression level of the hVEGF gene was measured by ELISA. The in vitro nuclear transport efficiency of the SV40-DTS plasmid was about $50 \%$ lower under hypoxia, while the HRE plasmid was about $50 \%$ higher under hypoxia. Quantitation of reporter gene expression in vitro and in vivo, under hypoxia and normoxia, confirmed that the SV40-DTS plasmid functioned better under normoxia, while the HRE plasmid was superior under hypoxia. These results indicate that the efficiency of gene expression by plasmids containing DNA binding sequences is affected by the concentration of oxygen in the medium.
\end{abstract}

Key words: SV40-DTS; Hypoxia-responsive element; Transfection efficiency; Normoxia; Hypoxia; Ischemic diseases

\section{Introduction}

Recent biotechnological advances have allowed the development of new technologies for the delivery of exogenous genetic material to mammalian cells. Functional genetic studies of these new technologies have resulted in a new therapeutic modality termed gene therapy. Of the several types of vectors available for gene therapy, naked DNA is used in $20 \%$ of current clinical trials (http://www.wiley. co.uk/genetherapy/clinical/). This non-viral vector is easy to produce, handle, and purify and generates a low immune response, allowing repeated administrations if necessary. Its utility in gene therapy, however, is currently limited by its low rate of transfection. Gene expression levels can be increased by facilitating plasmid entry into the nucleus (1), since the main barrier for plasmid vector transfection is the nuclear envelope crossing (2-5).

In 1997, Dean (1) demonstrated that plasmid nuclear import could be facilitated by the insertion into the plasmid of nuclear DNA-targeting sequences (DTS), which are transcription factor binding sites. The modified plasmid DNA is actively transported through nuclear membrane pores of non-dividing cells. In a subsequent study, this group isolated a 72-bp sequence from the Simian virus 40 (SV40) promoter containing binding sites for the NF-kappaB, Oct-1, SP1, SP2, AP1, and AP2 transcription factors $(4,6)$. The transcription factors are transported into the nucleus by importins and can carry the plasmid construct containing the SV40 nuclear DTS (SV40-DTS) with them (3-5). Other groups have successfully used DTS-containing plasmids. It was reported that the direct injection of the modified plasmid into the muscle tissue of rats increased gene expression 20-fold (7) and 10 -fold when injected into the blood vessel lumen (8) or when electroporated in mouse muscle cells (9).

The efficiency of the SV40-DTS sequence has been established by many studies under normoxia, but it has never been evaluated under hypoxia. Since the pattern of gene expression under hypoxia changes dramatically in

Correspondence: S.W. Han, UNIFESP-CINTERGEN, Rua Mirassol, 207, 04044-010 São Paulo, SP, Brasil. Fax: +55-11-5084-7582. E-mail: sang.han@unifesp.br

Received November 19, 2009. Accepted June 30, 2010. Available online July 8, 2010. Published August 13, 2010. 
response to the new physiological condition (10), the transcription factors used for plasmid transport must also shift. Consequently, it is unclear whether the vectors containing the SV40-DTS sequence will have the same efficiency of transfection under hypoxic condition. In terms of pathology, there are many diseases provoked by ischemia such as myocardial infarction, critical limb ischemia and stroke. For the first two diseases, plasmid-derived vectors have been widely used for clinical studies.

Under hypoxia, the hypoxia-inducible factor (HIF) is the main activated transcription factor and is responsible for the induction of expression of more than 50 genes related to hypoxia (11-13). The HIF is a heterodimeric protein with two subunits (alpha and beta) constitutively expressed by all cells of the body. Under hypoxic conditions, HIF-alpha subunits are no longer subjected to proteosomal degradation and are thereby able to dimerize with the beta subunits. The heterodimeric HIF activates transcription of genes carrying hypoxia-responsive elements (HRE), which are present in the promoter sequences of those genes $(11,12,14)$. Therefore, the HRE sequence is a good candidate for a DTS in plasmid vectors constructed for gene therapy of ischemic diseases.

The main objective of the present study was to find suitable DTS for the construction of plasmid vectors to be used to treat ischemic diseases. The well-known SV40-DTS and HRE sequences were used to construct plasmid vectors and the rate of plasmid nuclear transport and consequent gene expression under normoxia $\left(20 \% \mathrm{O}_{2}\right)$ and hypoxia (less than $5 \% \mathrm{O}_{2}$ ) were determined. Since the human vascular endothelial growth factor (hVEGF) gene has been extensively used to treat ischemic diseases, this gene was used as the reporter gene in our study.

\section{Material and Methods}

\section{Vector construction}

The backbone plasmid constructed for this study was named uP. For the uP vector construction, first the 600-bp cytomegalovirus (CMV) minimal promoter was removed from the pVAX1 vector (Invitrogen, USA) by Spel enzyme digestion (Figure 1). The complete immediate-early human CMV promoter from the gWIZ plasmid (Gene Therapy Systems, USA) was amplified by PCR with the oligonucleotides WIZSE (5'-AAACTAGTACCGCCATGTTGACATTG AT-3') and WIZAS (5'-TTACTAGTCGTGTCAAGGACGG TGACTG-3'), both containing 5' Spel hydrolysis sites. The PCR-amplified products were digested with Spel and inserted into the pVAX1 plasmid, previously digested with the same enzyme. The other vectors in this study were constructed from the uP backbone.

The SV40-DTS sequence (5'-ATGCTTTGCATACTT CTGCCTGCTGGGGAGCCTGGGGACTTTCCACACC CTAACTGACACACATTCCACAGCTGGTT-3' and its complementary sequence) $(4,7)$ and the HRE sequence (5'-GCC
CTACGTGCTGCCTCGCATGGCCCGGCTGACCTCTTG ACCCCTCTGGG-3' and its complementary sequence) (13) were synthesized (Invitrogen, Brazil), phosphorylated and annealed. For annealing, each pair of oligonucleotides was incubated at $95^{\circ} \mathrm{C}$ for $5 \mathrm{~min}$ in $50 \mathrm{mM} \mathrm{NaCl}$ and $1 \mathrm{mM}$ EDTA, pH 7.4. After slowly cooling to room temperature, the oligonucleotides were purified by precipitation with 0.3 M sodium acetate. These double-stranded oligonucleotides were then inserted into the EcoRV site of the uP vector to form the UPS (containing the SV40-DTS) or UPH (containing the HRE) vectors (Figure 1).

The hVEGF165 gene was cloned by RT-PCR from the human lymphoid cell line HL-60 with the oligonucleotides VEGFSE (5'-ATTGAATTCCGGGCCTCCGAAACCATG AAC-3') and VEGFAS (5'-TAAGGATCCAGGACTGTTCTG TCGATGGTG-3'). The RT-PCR products were phosphorylated, digested with EcoRI and ligated into the uP vectors that were previously digested with EcoRI and EcoRV (Figure 1). All new sequences were confirmed by DNA sequencing (ABI 3100-Avant, Applied Biosystems, Brazil).

\section{Cell culture, transfection and induction of hypoxia in vitro}

The human embryonic kidney cell line A293T was cultured in Dulbecco's modified Eagle's medium (DMEM; Invitrogen) supplemented with $2 \mathrm{mM}$ glutamine, $200 \mu \mathrm{g} / \mathrm{mL}$ streptomycin, $200 \mathrm{U} / \mathrm{mL}$ penicillin and $10 \%$ heat-inactivated fetal bovine serum (Invitrogen) and maintained at $37^{\circ} \mathrm{C}$ with $5 \% \mathrm{CO}_{2}$. Cell hypoxia was induced by the addition of cobalt chloride to a final concentration of $100 \mu \mathrm{M} 24$ h before transfection $(15,16)$, and this concentration was maintained throughout the experiment. Gene transfer was performed via co-precipitation of the UP-derived plasmids with calcium phosphate in $35-\mathrm{mm}$ plates containing $2.5 \times 10^{5}$ cells. Forty-eight hours after transfection, the supernatant was collected and used to determine the concentration of VEGF by ELISA.

\section{Induction of mouse limb ischemia}

Ten BALB/c mice (12-week-old males) from the animal house of the Universidade Federal de São Paulo (UNIFESP) were used in the experiments, which were carried out in accordance with the recommendations for the proper care and use of laboratory animals by the Ethics Committee of UNIFESP (approved project No. 842/04).

Mice were anesthetized with an intraperitoneal injection of ketamine and xylazine (40 and $10 \mathrm{mg} / \mathrm{kg}$ body weight, respectively). To induce limb ischemia, the deep and superficial femoral arteries were excised from their origin as a branch of the external iliac artery without damaging the femoralis vein or nervus, and were then ligated to the point where the femoral artery bifurcates into the saphenous and popliteal arteries. These last arteries and the circumflex artery were only ligated. Gene transfer was performed by injecting $100 \mu \mathrm{g}$ plasmid in $100 \mu \mathrm{L}$ PBS in the middle of 
the thigh with an insulin needle soon after the induction of ischemia. After the procedure, the animals were kept under analgesia with daily peritoneal injections of $5 \mathrm{mg} /$ $\mathrm{kg}$ carprofen. Forty-eight hours after gene transfer, the animals were euthanized by cervical displacement. The total mass of the thigh muscle was removed and frozen in liquid nitrogen. One milliliter of lysis solution $(25 \mathrm{mM}$ Tris- $\mathrm{HCl}, 50 \mathrm{mM} \mathrm{NaCl}, 0.5 \% \mathrm{Na}$-deoxycholate, 2\% NP-40, $0.2 \%$ SDS, $1 \mathrm{mM} \mathrm{PMSF}, 1 \mu \mathrm{g} / \mathrm{mL}$ aprotinin, and $1 \mu \mathrm{g} / \mathrm{mL}$ leupeptin) was added per $100 \mathrm{mg}$ tissue, which was then homogenized with the Ultra-Turrax T8 homogenizer (Ika, USA). The homogenate was centrifuged at $4500 \mathrm{~g}$ at $8^{\circ} \mathrm{C}$ for $30 \mathrm{~min}$ and the supernatant was used to determine protein concentration by the Bradford method (17) and VEGF by ELISA.

\section{Determination of VEGF concentration by ELISA}

To determine the level of VEGF protein expression by ELISA, 96-well plates were incubated with $1 \mu \mathrm{g} / \mathrm{mL}$ rabbit polyclonal anti-hVEGF antibody (Peprotech Inc., USA) at $4{ }^{\circ} \mathrm{C}$ overnight. After blocking with $3 \%$ BSA in PBS at room temperature for $1 \mathrm{~h}$, the samples diluted 2-fold in washing buffer $(0.1 \%$ BSA and $0.1 \%$ Tween-20 in PBS) were added to the 96 -well plate and incubated at $4^{\circ} \mathrm{C}$ for $2 \mathrm{~h}$. After four washes with washing buffer, the plates were incubated with $0.15 \mu \mathrm{g} / \mathrm{mL}$ biotin-conjugated rabbit anti-hVEGF antibody for $1 \mathrm{~h}$ at $37^{\circ} \mathrm{C}$ (Peprotech Inc.). Plates were washed four times and incubated for $30 \mathrm{~min}$ at room temperature with 1:1000-diluted streptavidin-horseradish peroxidase (Pierce Biotech, USA). After thorough washing, the reactions were developed by adding $3 \mathrm{mg} / \mathrm{mL}$ o-phenylenediamine diluted in $50 \mathrm{mM}$ citrate buffer and stopped with $4 \mathrm{~N} \mathrm{H}_{2} \mathrm{SO}_{4}$. The absorbance at $492 \mathrm{~nm}$ was measured with an EL 808 UltraMicroplate Reader (Bio-Tek Instruments, Inc., USA). Experiments were carried out at least in triplicate for each sample to obtain the mean and standard deviation.

\section{Cell nucleus isolation and quantitative real-time PCR}

For relative quantitation of plasmids using real-time quantitative PCR (Q-PCR), the A293T cells were plated and transfected as described above. Four hours later, the cells were harvested and incubated for $10 \mathrm{~min}$ in $10 \mathrm{mM} \mathrm{HEPES}$ buffer, pH 7.9, containing $10 \mathrm{mM} \mathrm{KCl}$ and $1 \mathrm{mM}$ EDTA for lysis of the plasma membrane. After isolation of the nuclei by centrifugation $(15,000 \mathrm{~g}, 3 \mathrm{~min})$, the QIAMP mini-kit (Qiagen, USA) was used for extraction and purification of DNA from the nuclei following manufacturer instructions. For Q-PCR, the SYBR Green PCR Master Mix (Applied Biosystems, Brazil) was used with $0.5 \mu \mathrm{M}$ of specific primers for each gene. Real-time PCR was performed on the ABI 7700 thermocycler with its software (Applied Biosystems). Reactions with primers for the glyceraldehyde 3-phosphate dehydrogenase gene (GAPDH) were used to normalize the results. The amplification conditions were: 1) one cycle at $95^{\circ} \mathrm{C}$ for $\left.10 \mathrm{~min}, 2\right) 35$ cycles at $94^{\circ} \mathrm{C}$ for $15 \mathrm{~s}, 60^{\circ} \mathrm{C}$ for 30 $\mathrm{s}$, and $72^{\circ} \mathrm{C}$ for $30 \mathrm{~s}$. The following primers were used for the amplifications: VEGF (5'-TTTCTGCTGTCTTGGGTG CATTGG and 5'-ACCACTTCGTGATTCTGCCCT) and GAPDH (5'-ACCACAGTCCATGCCATCAC and 5'-TCCA CCACCCTGTTGCTGTA).

For statistical analysis, the results for each experimental group were compared independently of the control group. Data are reported as means \pm SEM (standard error of the mean). The unpaired $t$-test was applied and $\mathrm{P}<0.05$ was considered to be significant in all experiments.

\section{Results and Discussion}

To understand the influence of DTS on the transport of plasmids to the nucleus under hypoxia and normoxia, A293T cells were first transfected with plasmid vectors (Figure 1). After $4 \mathrm{~h}$, a sufficient time for a plasmid to enter into the nucleus (18), whole nuclear DNA was isolated and used for quantitation of the plasmid concentration by Q-PCR. In cells transfected with the uPS-hVEGF plasmid, the nuclear plasmid concentration under normoxia was twice that observed under hypoxia (Figure 2). However, if the cells were transfected with the UPH-hVEGF vector this situation was reversed, with the concentration of plasmid in the nuclei under hypoxia being 2-fold higher than that found under normoxia (Figure 2). These statistically significant results suggest that the transport of DNA into the nucleus depends upon at least two factors: the type of DTS and the concentration of oxygen. The profiles of gene expression under normoxia and hypoxia are different, especially with the genes involved in oxygenation. Consequently, the DTS used for vector construction should be appropriate for each situation.

The control plasmid uP-hVEGF, which has no known DTS but contains the complete CMV intron A sequence, presented a statistically significant $30 \%$ reduction of nuclear plasmid concentration under hypoxia versus normoxia, much lower than differences seen with the vectors containing DTS (Figure 2). This result indicates that intron A weakly facilitates plasmid transport under hypoxia, perhaps through the binding of some unknown transcription factor activated under hypoxia. These results demonstrate a clear difference in the nuclear transport function of the SV40-DTS and HRE sequences under hypoxia and normoxia.

The final goal of designing a plasmid vector to treat ischemic diseases is to induce a high level of therapeutic gene expression under hypoxic condition. Efficient transport of the plasmid containing the HRE sequence (UPH-hVEGF) under hypoxia does not necessarily indicate that this vector will yield a high level of gene expression to produce a high concentration of VEGF protein by the transfected cells, because post-transcription and post-translation modifications and protein stability can influence protein determination. Therefore, it is fundamental to quantify protein expression from those transfected cells. 
In ELISA experiments with the uP-derived vectors under normoxia or hypoxia, VEGF gene expression obtained by the pVAX-hVEGF plasmid was used for normalization since its expression under normoxia or hypoxia did not show a significant variation (Figures 3 and 4). This result is expected because the expression of VEGF in this vector is under the control of only the CMV promoter and without no known DTS (19).

The profiles of VEGF gene expression in vitro for the
uP-derived vectors were very similar to the nuclear transport profiles (Figures 2 and 3); the rate of plasmid transport to the nucleus corresponded proportionally to the gene expression level, with some differences in absolute values. Cells transfected with the UPS-hVEGF plasmid produced twice the level of VEGF expression under normoxia than under hypoxia, while cells transfected with UPH-hVEGF tripled VEGF expression under hypoxia compared to normoxia (Figure 3). The data from uPS-hVEGF transfection under

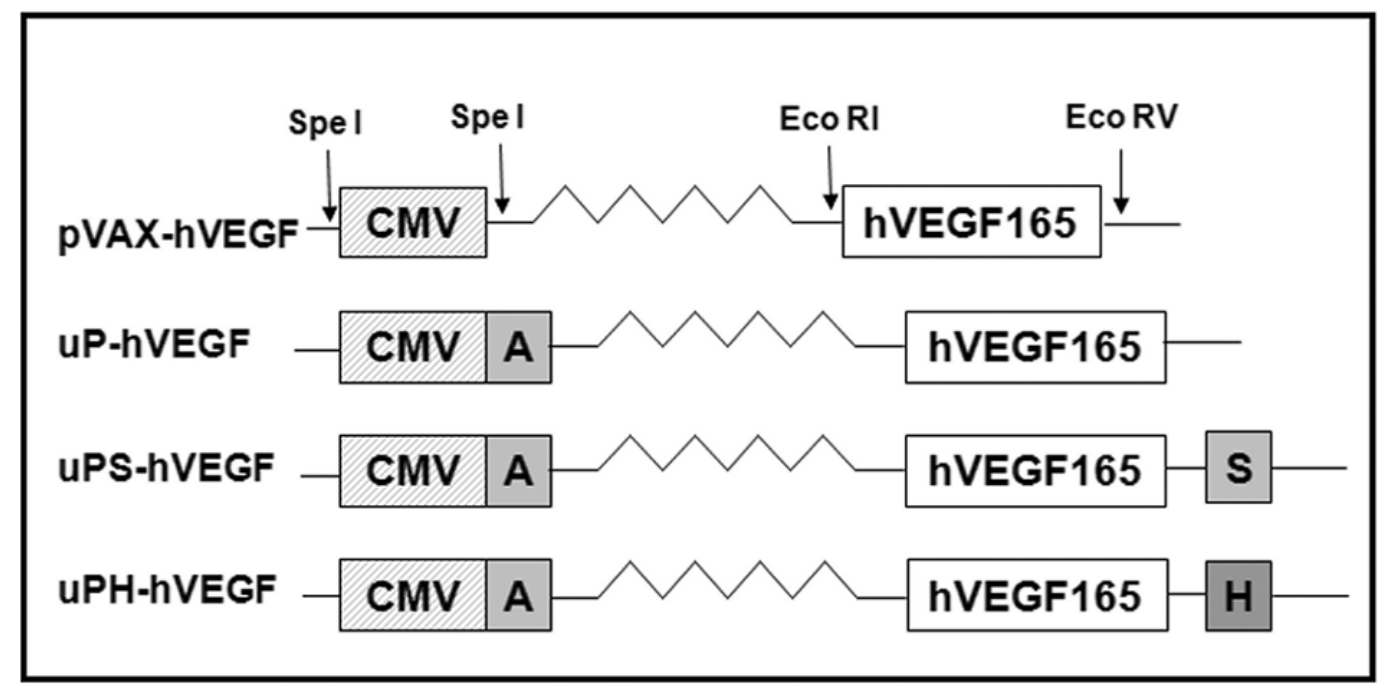

Figure 1. Schematic diagram of the plasmid. An Spel restriction site was used to replace the CMV minimal promoter from pVAX-hVEGF with CMV-A. An EcoRV restriction site was used to insert the $\mathrm{S}$ or $\mathrm{H}$ sequence in the uP-vascular endothelial growth factor 165 (uP-VEGF165) gene vector. S = SV40-DTS (SV40-DNA-targeting sequence); $\mathrm{H}=\mathrm{HRE}$ (hypoxia-responsive element); $\mathrm{A}=\mathrm{CMV}$ (cytomegalovirus) intron A.

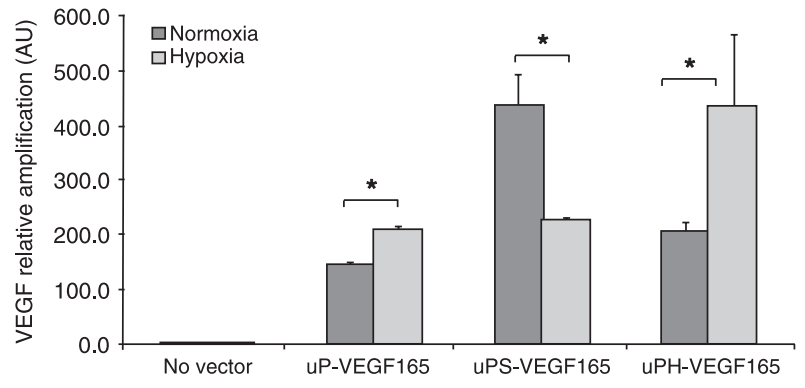

Figure 2. Q-PCR carried out to determine plasmid concentration in the nucleus. A293T cells were transfected with the indicated vectors and, after $4 \mathrm{~h}$, the nuclei were isolated and plasmids were purified to perform Q-PCR. The Q-PCR results were normalized using data from GAPDH amplification. The data are from four independent experiments. See Figure 1 for abbreviations. ${ }^{*} \mathrm{P}<$ 0.05 (unpaired $t$-test).

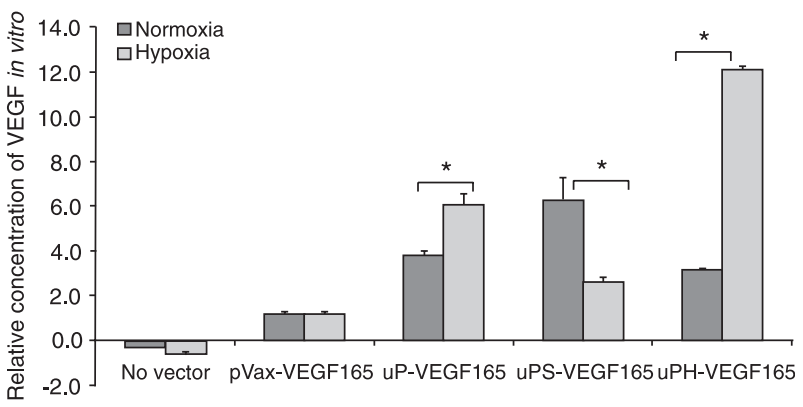

Figure 3. Vascular endothelial growth factor (VEGF) production from cells transfected with the indicated vectors. A293T cells were transfected and after $48 \mathrm{~h}$ VEGF concentration in the supernatant was determined by ELISA. The relative concentration of VEGF is reported using the pVAX-hVEGF gene expression under normoxia as one. Ten independent experiments were carried out at least in triplicate for each point to obtain the mean and standard deviation. See Figure 1 for abbreviations. ${ }^{*} \mathrm{P}<0.05$ (unpaired $t$-test). 
normoxia are consistent with previous results $(1,4)$, while results from the hypoxia experiments are not in agreement with previous results $(1,4)$ and support our hypothesis. The presence of the SV40-DTS sequence in the plasmid under hypoxia had no additional effect or could decrease gene expression (Figure 3), which is not desirable if one intends to treat ischemic diseases. In contrast, the high levels of gene expression under hypoxia and very low levels under normoxia observed with the UPH-hVEGF plasmid are the desired results after gene therapy to treat ischemic diseases. The production and action of angiogenic factors are required specifically by hypoxic tissues, and those factors can provoke pathological angiogenesis, such as tumors, in normoxic tissues. Our results from this in vitro study clearly demonstrate different levels of gene expression under different oxygen concentrations for plasmids containing the SV40-DTS versus HRE sequences.

In the in vivo study, plasmids were injected into the mouse quadriceps and the VEGF concentration was determined after $48 \mathrm{~h}$. Muscle crude extract rather than blood was used for quantification because most VEGF remains at the local site of production due to the heparin-binding domain of the VEGF molecule (20). Therefore, the concentration of VEGF in the blood was very low and sometimes could not be detected (data not shown).

The trend of gene expression seen in vitro could be reproduced in vivo on a much smaller scale, but the variation of gene expression was not statistically significant for most conditions, even when using about eight animals per group (Figure 4). One of the reasons was the high endogenous VEGF gene expression caused by the ischemic surgery (Figure 4). In addition, variations during in vivo experiments are frequently large $(7,19,21,22)$ and probably a larger number of animals per group could reduce the standard error of the mean, which could be sufficient to demonstrate a statistically significant change in gene expression. However, the gene expression resulting from transfection with the UPH-hVEGF plasmid was markedly different from that obtained with other vectors and conditions: under ischemic conditions it promoted a 10-fold increase in the expression compared to normoxia (Figure 4). Even compared to the UP-hVEGF plasmid under hypoxia, expression from the UPH-hVEGF plasmid was 2-fold higher. This result is consistent with earlier discussions, which demonstrate that the presence of the HRE sequence in the plasmid has a

\section{References}

1. Dean DA. Import of plasmid DNA into the nucleus is sequence specific. Exp Cell Res 1997; 230: 293-302.

2. Dean DA, Strong DD, Zimmer WE. Nuclear entry of nonviral vectors. Gene Ther 2005; 12: 881-890.

3. Wilson GL, Dean BS, Wang G, Dean DA. Nuclear import of plasmid DNA in digitonin-permeabilized cells requires both cytoplasmic factors and specific DNA sequences. $J$ Biol

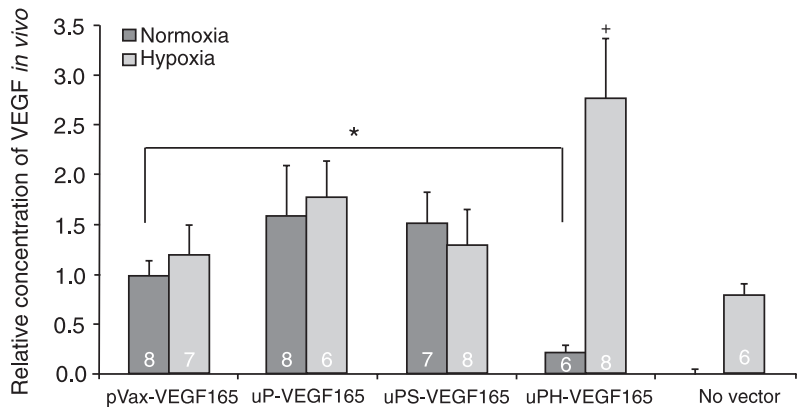

Figure 4. In vivo vascular endothelial growth factor (VEGF) production after transfection with the indicated vectors. ELISA was performed using a crude extract from the mouse thigh muscle 48 $\mathrm{h}$ after transfection. The numbers of animals used for each experiment are indicated in the columns, and at least triplicate assays were carried out for each point to obtain the mean and standard deviation. The relative concentration of VEGF is reported using the pVAX-hVEGF gene expression under normoxia as one. See Figure 1 for abbreviations. To compare all groups Kruskal-Wallis test $\left({ }^{*}\right)$ was applied and Dunn's post test $(+)$ was used to compare two columns. Only $P$ values $<0.05$ were considered to be statistically significant.

stronger effect under hypoxia, a result not observed with the vector containing the SV40-DTS (Figure 4).

The uPH-hVEGF plasmid expressed less than pVAXhVEGF under normoxia, opposing the previous result from the in vitro study (Figure 3). We have no explanation for this, but we suspect that this was caused by one or more unknown factors present in skeletal muscle cells that have affinity for the DTS present in the UPH-hVEGF plasmid.

Our findings indicate that the inappropriate use of the SV40-DTS or HRE sequences in the construction of plasmid vectors can strongly affect the expression of heterologous genes and compromise the expected therapeutic effect of these plasmids. Therefore, vectors should be evaluated under physiologically relevant conditions before their application to gene therapy.

\section{Acknowledgments}

Research supported by FAPESP (\#06/59630-0). C.B. Sacramento was the recipient of a scholarship from CNPq. 
6. Kang HJ, Kim HS, Zhang SY, Park KW, Cho HJ, Koo BK, et al. Effects of intracoronary infusion of peripheral blood stemcells mobilised with granulocyte-colony stimulating factor on left ventricular systolic function and restenosis after coronary stenting in myocardial infarction: the MAGIC cell randomised clinical trial. Lancet 2004; 363: 751-756.

7. Li S, MacLaughlin FC, Fewell JG, Gondo M, Wang J, Nicol $F$, et al. Muscle-specific enhancement of gene expression by incorporation of SV40 enhancer in the expression plasmid. Gene Ther 2001; 8: 494-497.

8. Young JL, Benoit JN, Dean DA. Effect of a DNA nuclear targeting sequence on gene transfer and expression of plasmids in the intact vasculature. Gene Ther 2003; 10: 1465-1470.

9. Blomberg P, Eskandarpour M, Xia S, Sylven C, Islam KB. Electroporation in combination with a plasmid vector containing SV40 enhancer elements results in increased and persistent gene expression in mouse muscle. Biochem Biophys Res Commun 2002; 298: 505-510.

10. Greijer AE, van der Groep P, Kemming D, Shvarts A, Semenza GL, Meijer GA, et al. Up-regulation of gene expression by hypoxia is mediated predominantly by hypoxia-inducible factor 1 (HIF-1). J Pathol 2005; 206: 291-304.

11. Pugh CW, Ratcliffe PJ. Regulation of angiogenesis by hypoxia: role of the HIF system. Nat Med 2003; 9: 677-684.

12. Haddad JJ, Harb HL. Cytokines and the regulation of hypoxia-inducible factor (HIF)-1alpha. Int Immunopharmacol 2005; 5: 461-483.

13. Semenza GL, Wang GL. A nuclear factor induced by hypoxia via de novo protein synthesis binds to the human erythropoietin gene enhancer at a site required for transcriptional activation. Mol Cell Biol 1992; 12: 5447-5454.
14. Jiang BH, Rue E, Wang GL, Roe R, Semenza GL. Dimerization, DNA binding, and transactivation properties of hypoxiainducible factor 1. J Biol Chem 1996; 271: 17771-17778.

15. Miyazaki K, Kawamoto T, Tanimoto K, Nishiyama M, Honda $\mathrm{H}$, Kato $\mathrm{Y}$. Identification of functional hypoxia response elements in the promoter region of the DEC1 and DEC2 genes. $J$ Biol Chem 2002; 277: 47014-47021.

16. Yuan Y, Hilliard G, Ferguson T, Millhorn DE. Cobalt inhibits the interaction between hypoxia-inducible factor-alpha and von Hippel-Lindau protein by direct binding to hypoxia-inducible factor-alpha. J Biol Chem 2003; 278: 15911-15916.

17. Bradford MM. A rapid and sensitive method for the quantitation of microgram quantities of protein utilizing the principle of protein-dye binding. Anal Biochem 1976; 72: 248-254.

18. Satkauskas S, Bureau MF, Mahfoudi A, Mir LM. Slow accumulation of plasmid in muscle cells: supporting evidence for a mechanism of DNA uptake by receptor-mediated endocytosis. Mol Ther 2001; 4: 317-323.

19. Lee Y, Park EJ, Yu SS, Kim DK, Kim S. Improved expression of vascular endothelial growth factor by naked DNA in mouse skeletal muscles: implication for gene therapy of ischemic diseases. Biochem Biophys Res Commun 2000; 272: 230235.

20. Cross MJ, Dixelius J, Matsumoto T, Claesson-Welsh L. VEGF-receptor signal transduction. Trends Biochem Sci 2003; 28: 488-494.

21. Luo F, Wariaro D, Lundberg G, Blegen H, Wahlberg E. Vascular growth factor expression in a rat model of severe limb ischemia. J Surg Res 2002; 108: 258-267.

22. Shyu KG, Chang H, Wang BW, Kuan P. Intramuscular vascular endothelial growth factor gene therapy in patients with chronic critical leg ischemia. Am J Med 2003; 114: 85-92. 